\title{
I Want to Control Your Move: Human- Human Interface (HHI) Neuromuscular Electrical Stimulator (NMES)
}

\author{
Ching Yee YONG ${ }^{1}$ and Terence Tien Lok SIA \\ School of Engineering and Technology, University College of Technology Sarawak, \\ Sibu, Sarawak, Malaysia
}

\begin{abstract}
Neuromuscular electrical stimulation (NMES) has been widely used in rehabilitation hubs to restore or replace the motor function of individuals who have upper neuron damage such as stroke and spinal cord injury. However, the utilization of sensors in NMES is limited and results in the lack of data for upper limb movement analysis. The proposed system implemented NMES integrated with human-to-human interface (HHI) in the rehabilitation process for stroke patients. The therapist (controller) can coach the motion of patients (subject) by injecting his own signal for patients to follow. Ten (10) subjects were tested with five (5) repeating trials. The EMG value was extracted from the finger flexion and extension at the controller side, then injected into the control unit for further stimulation of the subject. In order to evaluate the repeating motion by the subject, an accelerometer was attached to the finger. Performance evaluation of the subject was executed by comparing the flexion angle with the controller side. The result showed that the error of the system was less than $10.29 \%$ for the first trial and gradually reduced to $1 \%$ after 5 trials.
\end{abstract}

Keywords. Accelerometer, EMG, human-human interface (HHI), motion, neuromuscular electrical stimulation (NMES), home-based rehabilitation

\section{Introduction}

The rehabilitation for stroke patients can help them relearn skills that were lost when a stroke affected a part of their brains. Normally, the patients will undergo rehabilitation exercises through physical therapy to strengthen the muscles [1]. Unlike the patients with significant locomotors impairment, the gait analysis was introduced as the therapist's clinical tool for analyzing the walking pattern of the patients. Normally, the physician carries out an observational gait assessment to identify the specific gait dysfunctions of patients through the practice routine [2]. The physician provides useful information and guides the patients in locomotors training by using video images or the naked eye in observing the whole gait analysis [3]. However, the observational gait assessment has lacked in studying the psychometric properties. This study [4] shows that physical therapists can give an accurate rating of gait deviation when the task is clearly defined and with a single parameter to judge. The observational gait assessment has limited study in psychometric properties. The physical therapists can give an

${ }^{1}$ Corresponding Author, Ching Yee YONG, School of Engineering and Technology, University College of Technology Sarawak, Sibu, Sarawak, Malaysia; E-mail: ching.yee@ucts.edu.my. 
accurate rating of gait deviation when the task is clearly defined and with a single parameter to judge [5]. The home-based rehabilitation program allows the patients to perform NMES at home, thus help them to regain independence in performing daily activities. The result shows that the performance of the patient can be dependent on the physiotherapist by providing the performance feedback to correct the patients' error [6]. In sports science, the process of the motion assessment can be done by utilizing the diagnostic tools or sensors to provide measurable information in coaching the athletes in performing exercise programs [7]. The data has been an important factor in this era which may help to analyze, create new strategies and thus boost a player's performance. The real-time performance is targeted for monitoring the performance of an athlete which may avoid muscle fatigue [8].

NMES can be used as a motor relearning tool to let the patients participate in goaloriented repetitive movement therapy [9]. This kind of improvement in upper limb function using the NMES device can be called a therapeutic effect. Moreover, the patients who are in the chronic phase of the stroke where the motor relearning strategies do not work against it [10]. NMES can be used as a neuroprosthesis with the primary intent to let the patients execute functional tasks with the affected upper limb while using the device as part of routine daily living. This improvement on the affected upper limb while using the NMES device is called the neuro-prosthetic effect [11].

Knutson et al. [12] proposed a method of electrical stimulation which is contralaterally controlled functional electrical stimulation (CCFES) to help improve recovery of volitional hand function for patients with hemiplegic stroke. This method provides the patient with the opportunity to control the stimulation of the paretic hand in realtime by opening and closing the non-paretic hand. On the non-paretic hand side, it is worn with an instrumented glove that is able to modulate the stimulation intensity to the paretic hand by detecting the degree of hand opening. Ambrosini et al. [13] proposed the system with a multi-joint robot and NMES hybrid system for the coordinated upper limb physical practice at the elbow, wrist, and fingers. The proposed system is a wearable device that supports the patients to perform sequencing limb movements such as elbow extension, wrist extension associated with hand open, wrist flexion, and elbow flexion.

Rong et al. [14] proposed the closed-loop control system which is an EMGcontrolled neuroprosthesis to exploit maximally the user contribution during hand-tomouth movement with the help of a passive exoskeleton for weight support. The intensity of the volitional muscle activity from EMG data is then measured at the stimulation period of $50 \mathrm{~ms}$. The EMG signal is generated where further compared to the preset threshold to trigger the electrical stimulation. Cunningham proposed the use of contra-laterally controlled functional electrical stimulation (CCFES) to help to improve the recovery of volitional hand function for patients with hemiplegic stroke [15]. Cunningham proposed a closed-loop control system which is an EMG-controlled neuroprosthesis to exploit maximally the user's contribution during hand-to-mouth movement with the help of passive technology.

The stimulation intensity refers to the current where its amplitude can vary from 0 to $140 \mathrm{~mA}$ for some ES devices [6]. Muscle contraction required high intensity during ES where the maximal contraction is not focusing in most ES applications. This is because the ES only activates the small portion of motor units underneath the surface electrode which does not contribute much in producing maximal contraction. At this point, the level of discomfort will increase as increasing the intensity. Furthermore, the intensity required to achieve the desired contraction is varied among the patients 
according to the individual sensation, skin resistance, size of muscle and size of electrodes. The contact of electrodes with the skin in different sessions will result in variation of the intensity provided. Nevertheless, the intensity may need to be increased during the session to adjust for accommodation.

The existing system for NMES only has a preset value of the stimulation parameter at which the patients cannot control the stimulation intensity. The proposed system integrated $\mathrm{HHI}$ as the communication channel to control the upper limb movement of patients. Furthermore, sensors are deployed in detecting flexion angle to control the stimulation intensity. Since the NMES is applied to the patient's skin, the safety of the patient is an essential consideration in designing the output circuit of NMES. Galvanic isolation was done for the H-bridge topology and transformer.

\section{Materials and Methods}

Figure 1 depicted the flow of the study. A pushbutton was included in the design in order to ensure continuous data uploading to the cloud after the WiFi module was activated. After this step was taken, the system will automatically perform the following such as getting the input from the controller and trigger the same strength to the subject's muscle. All the data was uploaded into the cloud for storage and further analysis by a professional practitioner. The subject can monitor his/her progress through the LED indicators.

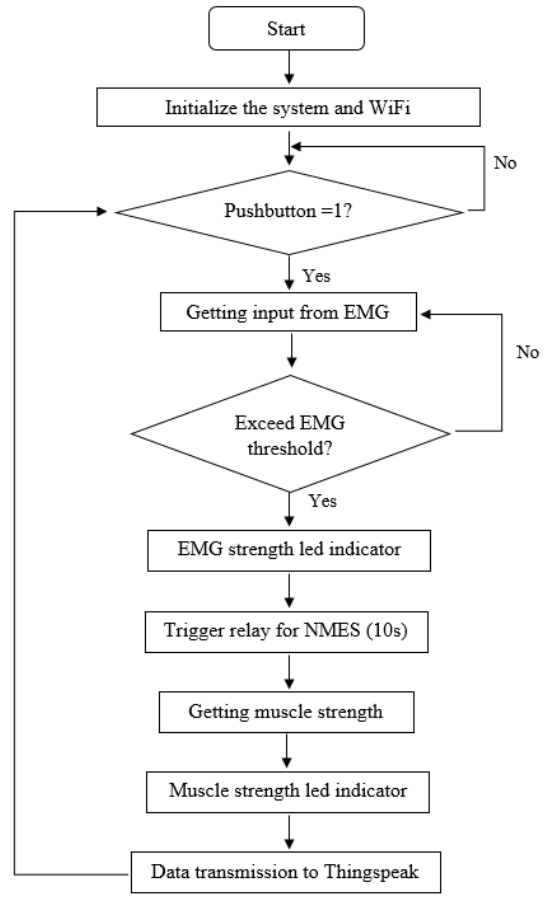

Figure 1. Methodology flow chart.

Ten (10) individuals (male and female) with the ages of 21-25 years were recruited in the study. The subjects are healthy and without joint issues in the upper limb and do 
not encounter any significant soreness in the flexor carpi ulnaris during the participation.

The HHI required both the controller (physiotherapist) and the subject (patient) to complete the NMES process. On the controller side, two of the surface electrodes which are red and black for the EMG circuit were placed at the forearm and dorsum of the hand respectively. On the other hand, the surface electrodes were positioned at the forearm and attached at the top of the ulnar nerve which is directly connected to the little finger and the adjacent half of the ring finger. The end of the surface electrodes is connected to a signal stimulator to produce electrical stimulation for fingers' movement observation. Two (2) electrodes were used to deliver the pulses of electrical current. The accelerometer sensor was attached to the subject's finger with elastic bands to obtain the movement values. Figure 2 shows the proposed prototype unit.

The frequency and pulse width of the stimulation signals were set for constant at $120 \mathrm{~Hz}$ and $110 \mu \mathrm{s}$. Meanwhile, the level of intensity (amplitude of signal release to the subject) was set to 2 for the initial condition. The subject's hand was placed parallel to the table to obtain a $0^{\circ}$ at the z-axis initially. 12 LEDs located at the top of the prototype were used to indicate muscle strength. 6 LEDs represent different strengths for the controller and subject respectively. The EMG value represents the muscle strength produced from the controller side while the accelerometer sensor values represent the muscle strength produced from the finger movement by the subject. The result will be displayed through 6 LEDs where each led represents a specific range of values.

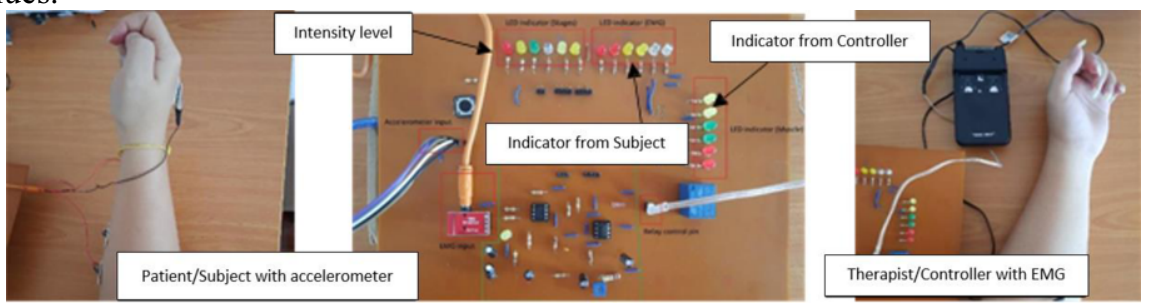

Figure 2. Controller set-up (left), HHI based NMES prototype (center), Subject set-up (right).

The EMG detects the electrical signals transmitted from the motor neuron when there are muscle contraction activities. This electrical signal is referred to as the analog signal, whereas it needs to be converted to a digital signal by using the analog to digital converter (ADC) for further analysis. The collected data is further used to determine signal strength from the controller's elbow movement and angle of elbow flexion produced during NMES. The proposed system examined the EMG signal from the controller elbow flexion and checked it with the EMG threshold. When the EMG threshold is exceeded, the relay is triggered ON to perform NMES with a duration of 10 seconds at the controlled person's hand. The muscle movement is detected using an accelerometer sensor with reading. The EMG signal level and muscle movement level for a controlled person hand are both shown by the led indicator. The proposed system collects four types of data which are EMG signal level, muscle movement level, EMG trigger value, and the muscle movement reading at the z-axis. The data is transmitted to the Thingspeak through a WiFi shield with a time of approximately 15 seconds per data. 


\section{Results and Discussion}

Table 1 shows the experimental results of 10 subjects for the first trial. The process was repeated 5 times for every subject. When the intensity level was increased (stimulation signals), the error was reduced. The percentage error of each subject was computed by the formula as follow:

$$
\text { error }(\%)=\frac{E M G \text { controller }-E M G \text { subject }}{E M G \text { controller }} \times 100 \%
$$

Table 1. Data collection and experimental results for the first trial

\begin{tabular}{ccccccc}
\hline \multirow{2}{*}{ Subject } & $\begin{array}{c}\text { Intensity } \\
\text { Level }\end{array}$ & \multicolumn{2}{c}{$\begin{array}{c}\text { Indicator (Controller) } \\
\text { LED }\end{array}$} & EMG & \multicolumn{2}{c}{ Indicator (Subject) } \\
LED & EMG & Error (\%) \\
\hline 1 & 2 & 5 & 349 & 5 & 336 & 3.725 \\
2 & 3 & 5 & 340 & 5 & 334 & 1.765 \\
3 & 3 & 5 & 330 & 5 & 327 & 0.909 \\
4 & 3 & 5 & 342 & 5 & 338 & 1.170 \\
5 & 3 & 5 & 340 & 5 & 332 & 2.353 \\
6 & 3 & 6 & 350 & 5 & 346 & 1.143 \\
7 & 2 & 6 & 350 & 5 & 314 & 10.286 \\
8 & 2 & 6 & 350 & 5 & 338 & 3.429 \\
9 & 2 & 6 & 360 & 5 & 332 & 7.778 \\
10 & 2 & 6 & 350 & 5 & 330 & 5.714 \\
$\vdots$ & $\vdots$ & $\vdots$ & $\vdots$ & $\vdots$ & $\vdots$ & $\vdots$ \\
\hline
\end{tabular}

Figure 3 shows the mechanism of the proposed stimulator. The design was divided into LED and sensors parts. The patient can monitor their progress at home by observing the illumination of the LED lights. The progress can be categorized as satisfactory if the number of LED lit up by the subject is the same as the controller's.

The collected data from the sensors are purposely for a physiotherapist for progress analysis. The professional therapist can monitor and analyze the patient/user progress by analyzing the numerical values. The user may perform the rehab activity at home since all the data will be uploaded online in real-time automatically. The therapist can retrieve the data at anytime, anywhere through a PC or a mobile phone. The main goal of this project is to design a portable IoT gadget for rehabilitation purposes. The error was between $0.91 \%$ and $10.29 \%$ at the first trial. This error can be related to the skin impedance, fat tissue of an individual or misplacement of an electrode at the stimulation area. The error was gradually reduced after several trials.

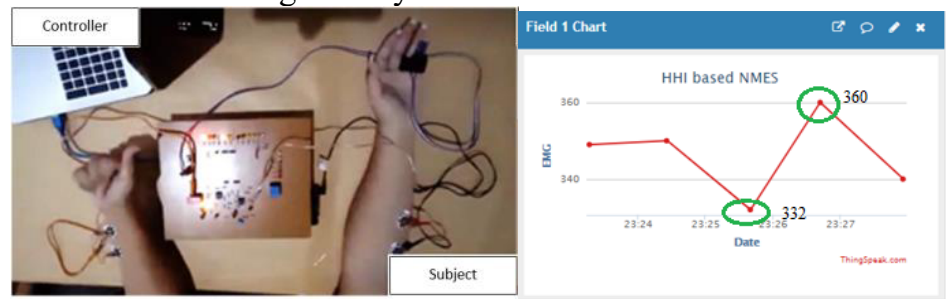

Figure 3. The controller is injecting the motion signal to the NMES (left), the IoT platform.

\section{Conclusion and Future Work}

The proposed system can be used in the rehabilitation hub for physiotherapy sessions. The HHI required the physiotherapist to take the role of the controller while the patients are being coached for motion. Furthermore, the therapist can control the 
stimulation timing through the push button on the board. The therapist and the patients can also monitor the stimulation result by observing the LED indicators and sensors values. The LED indicators will show the result based on the capability of the patients to perform the same action as the therapist does. Besides, the data of the stimulation progress is uploaded and stored in the cloud for further analysis. Thingspeak serves as the IoT platform where the data of each patient can be visualized and analysed in the cloud. The therapist can easily access this app through mobile or website to monitor the data.

Future work will cover the implementation of fuzzy logic using MATLAB to achieve digital control of triggering the electrical stimulation based on the EMG value change at the controller side. The EMG value, angle of finger flexion and extension are used as the input linguistic variable while the stimulation intensity is used as the output linguistic variable. Through this fuzzy logic model, the stimulation intensity is varied based on the rule set to achieve automation control for both controller and subject sides.

\section{Ethical Clearance and Conflict of Interest}

This study was reviewed ethically according to the code of ethical principles and standards. Written informed consent forms were sent out to the participants and parents for consent approval. The collected information has complied with Data Protection Regulations, and not disclosed to an external party. Standard operating procedures were followed in promoting the best possible ethical research practice. The authors declare no conflict of interest. Gratitude to the University College of Technology Sarawak.

\section{References}

[1] Arip, Eza SM, Waidah I, Md JN, Abduljalil R. Virtual reality rehabilitation for stroke patients: recent review and research issues. In AIP Conference Proceedings. 1905(1): p. 050007. 2017.

[2] Ridao-Fernández C, Pinero-Pinto E, Chamorro-Moriana G. Observational gait assessment scales in patients with walking disorders: systematic review. BioMed research international. 2019.

[3] Yong CY, Sudirman R, Chew KM. Motion detection and analysis with four different detectors. Third International Conference on Computational Intelligence, Modelling \& Simulation; 2011. p. 46-50.

[4] Nadeau S, Martina B, Francois B. Gait analysis for poststroke rehabilitation: the relevance of biomechanical analysis and the impact of gait speed. Physical Medicine and Rehabilitation Clinics. 2013; 24(2): 265-276.

[5] Naili JE, Anna-Clara E, Maura DI, Michael HS, Margareta H, Charlotte KH, Eva WB. The impact of symptomatic knee osteoarthritis on overall gait pattern deviations and its association with performancebased measures and patient-reported outcomes. The Knee. 2017; 24(3):536-546.

[6] Stevens-Lapsley JE, Jaclyn EB, Pamela W, Donald GE, Robert SS, Margaret S, Wendy MK. Relationship between intensity of quadriceps muscle neuromuscular electrical stimulation and strength recovery after total knee arthroplasty. Physical therapy. 2012; 92(9):1187-1196.

[7] Seshadri DR, Magliato S, Voos JE, Drummond C. Clinical translation of biomedical sensors for sports medicine. Journal of medical engineering \& technology. 2019; 43(1):66-81.

[8] Kim H, Jongho L, Jaehyo K. Electromyography-signal-based muscle fatigue assessment for knee rehabilitation monitoring systems. Biomedical engineering letters. 2018; 8(4):345-353.

[9] Cano-De-La-Cuerda R, Molero-Sánchez A, Carratalá-Tejada M, Alguacil-Diego IM, Molina-Rueda F, Miangolarra-Page JC, Torricelli D. Theories and control models and motor learning: clinical applications in neurorehabilitation. Neurología. 2015; 30(1):32-41.

[10] Li S. Spasticity, motor recovery, and neural plasticity after stroke. Frontiers in neurology. 2017; 8(120).

[11] Knutson J., Mary YH, Terri ZH, John C. Improving hand function in stroke survivors: a pilot study of contralaterally controlled functional electric stimulation in chronic hemiplegia. Archives of physical medicine and rehabilitation. 2007; 88(4):513-520. 
[12] Knutson JS, Michael JF, Lynne RS, John C. Neuromuscular electrical stimulation for motor restoration in hemiplegia. Physical medicine and rehabilitation clinics of North America. 2015; 26(4):729.

[13] Ambrosini E, Simona F, Marta T, Thomas S, Christian K, Giancarlo F, Alessandra P. An EMGcontrolled neuroprosthesis for daily upper limb support: a preliminary study. 2011 Annual International Conference of the IEEE Engineering in Medicine and Biology Society; 2011 p. 4259-4262.

[14] Wei R, Li W, Pang M, Hu J, Wei X, Yang B, Wai H, Zheng X, and Hu X. A Neuromuscular Electrical Stimulation (NMES) and robot hybrid system for multi-joint coordinated upper limb rehabilitation after stroke." Journal of neuroengineering and rehabilitation. 2017; 14(1):1-13.

[15] Cunningham DA, Jayme SK, Vishwanath S, Kelsey AP, Andre GM, Ela BP. Bilateral Contralaterally Controlled Functional Electrical Stimulation Reveals New Insights Into the Interhemispheric Competition Model in Chronic Stroke. Neurorehabilitation and neural repair. 2019; 33(9): 707-717. 